\title{
Circulant $m$-Diagonal Matrices Associated with Chebyshev Polynomials
}

\author{
Ahmet Öteleş \\ Department of Mathematics, Faculty of Education, Dicle University, Diyarbakir, Turkey
}

\section{Article Info}

Keywords: Chebyshev polynomial, Circulant matrix, Eigenvalue, Eigenvector 2010 AMS: 15Bxx, 15A18, $12 E 10$

Received: 13 October 2020

Accepted: 14 March 2021

Available online: 15 March 2021

\begin{abstract}
In this study, we deal with an $m$ banded circulant matrix, generally called circulant $m$ diagonal matrix. This special family of circulant matrices arise in many applications such as prediction, time series analysis, spline approximation, difference solution of partial differential equations, and so on. We firstly obtain the statements of eigenvalues and eigenvectors of circulant $m$-diagonal matrix based on the Chebyshev polynomials of the first and second kind. Then we present an efficient formula for the integer powers of this matrix family depending on the polynomials mentioned above. Finally, some illustrative examples are given by using maple software, one of computer algebra systems (CAS).
\end{abstract}

\section{Introduction}

Multiplying a vector by a circulant matrix is equivalent to a well-known operation called a circular convolution. Convolution operations, and so circulant matrices, arise in number of applications: digital signal processing, image compression, physics/engineering simulations, number theory, coding theory, cryptography, etc. Numerical solutions of certain types of elliptic and parabolic partial differential equations with periodic boundary conditions often involve linear systems associated with circulant matrices [1]-[3].

A certain type of transformation of a set of numbers can be represented as the multiplication of a vector by a square matrix. Repetition of the operation is equivalent to multiplying the original vector by a power of the matrix. Solving some difference equations, differential and delay differential equations and boundary value problems, we need to compute the arbitrary integer powers of a square matrix $[4,5]$. The powers of matrices are thus of considerable importance.

Computing the integer powers of circulant matrices depending on Chebyshev polynomials recently has been a very attractive problem [6]-[13]. For example, Rimas obtained a general expression for the entries of the $r^{\text {th }}$ power $(r \in \mathbb{N})$ of the $n \times n$ real symmetric circulant $\operatorname{circ}_{n}(0,1,0, \ldots, 0,1)$ (see [6] or [7] for the odd case and [8] or [9] for the even case). In [10], Gutiérrez obtained a general expression for the entries of the positive integer powers of complex symmetric circulant matrix given by

$$
\begin{array}{ll}
\operatorname{circ}_{n}\left(b_{0}, b_{1}, \ldots, b_{\frac{n-1}{2}}, b_{\frac{n-1}{2}}, \ldots, b_{1}\right) & \text { if } n \text { is odd } \\
\operatorname{circ}_{n}\left(b_{0}, b_{1}, \ldots, b_{\frac{n}{2}-1}, b_{\frac{n}{2}}, b_{\frac{n}{2}-1}, \ldots, b_{1}\right) & \text { if } n \text { is even }
\end{array}
$$

by generalizing the results derived by Rimas in [6]-[9].

In [11], Köken et al. obtained a general expression for the entries of the $r^{t h}$ power $(r \in \mathbb{N})$ of odd order circulant matrices of the type $\operatorname{circ}_{n}(0, a, 0, \ldots, 0, b)$. In [12], we presented a single expression for the integer powers of the circulant matrix $\operatorname{circ}_{n}\left(a_{0}, a_{1}, 0, \ldots, 0, a_{-1}\right)$ of odd and even order by generalizing the results derived by Köken et al. in [11]. 
In the current study, we consider an $n \times n$ circulant $m$-diagonal matrix $A_{n}$, that clearly is as,

$$
\begin{aligned}
& A_{n}=\operatorname{circ}_{n}\left(a_{0}, a_{1}, \ldots, a_{m}, 0, \ldots, 0, a_{-m}, \ldots, a_{-1}\right) \\
& =\left[\begin{array}{cccccccccc}
a_{0} & a_{1} & \cdots & a_{m} & 0 & \cdots & 0 & a_{-m} & \cdots & a_{-1} \\
a_{-1} & a_{0} & a_{1} & \ddots & a_{m} & 0 & \ddots & \ddots & \ddots & \vdots \\
\vdots & a_{-1} & a_{0} & a_{1} & \ddots & \ddots & \ddots & \ddots & \ddots & a_{-m} \\
a_{-m} & \ddots & a_{-1} & a_{0} & \ddots & \ddots & \ddots & \ddots & \ddots & 0 \\
0 & a_{-m} & \ddots & \ddots & \ddots & \ddots & \ddots & \ddots & 0 & \vdots \\
\vdots & 0 & \ddots & \ddots & \ddots & \ddots & \ddots & \ddots & a_{m} & 0 \\
0 & \ddots & \ddots & \ddots & \ddots & \ddots & \ddots & \ddots & \ddots & a_{m} \\
a_{m} & \ddots & \ddots & \ddots & \ddots & \ddots & \ddots & \ddots & a_{1} & \vdots \\
\vdots & \ddots & \ddots & \ddots & 0 & a_{-m} & \cdots & a_{-1} & a_{0} & a_{1} \\
a_{1} & \cdots & a_{m} & 0 & \cdots & 0 & a_{-m} & \cdots & a_{-1} & a_{0}
\end{array}\right]
\end{aligned}
$$

for all $3 \leq n \in \mathbb{N}$, where $1 \leq m \leq\left\lfloor\frac{n-1}{2}\right\rfloor$ and

$$
a_{-i}=a_{n-i}, \quad i=1, \ldots, m \text {. }
$$

We organize this paper as the following parts. In Section 2, we give some fundamental notations, definitions and important properties that we will need for the next sections. In Section 3, we introduce Lemma 3.1 and Theorem 3.3 that respectively give the statements of eigenvalues and eigenvectors of circulant $m$-diagonal matrix in (1.2) depending on the Chebyshev polynomials of the first and second kind. In Section 4, we obtain an efficient expression for the integer power of this matrix by means of the polynomials mentioned above. In Section 5, some illustrative examples are given. Finally, we will finish the paper with two Maple procedures.

\section{Preliminaries}

In this part, we present some fundamental notations, definitions and necessary properties for the next parts. An $n \times n$ circulant matrix is defined in [14] as

$$
C_{n}:=\left[\begin{array}{cccccc}
c_{0} & c_{1} & c_{2} & \ldots & c_{n-2} & c_{n-1} \\
c_{n-1} & c_{0} & c_{1} & \ddots & & c_{n-2} \\
c_{n-2} & c_{n-1} & c_{0} & \ddots & \ddots & \vdots \\
\vdots & \ddots & \ddots & \ddots & \ddots & c_{2} \\
c_{2} & & \ddots & \ddots & \ddots & c_{1} \\
c_{1} & c_{2} & \ldots & c_{n-2} & c_{n-1} & c_{0}
\end{array}\right]
$$

where $c_{i j}=c_{(j-i)(\bmod n)}$. It can be clearly seen from above that each row of $C_{n}$ is a cyclic shift of the previous row. Since $C_{n}$ has at most $n$ distinct elements in each row, it is often represented by

$$
C_{n}:=\operatorname{circ}_{n}\left(c_{0}, c_{1}, \ldots, c_{n-1}\right) .
$$

Let $n \geq 1$ be a fixed integer and $\omega$ be the primitive $n$th root of unity; namely, $\omega=e^{\mathbf{i} \frac{2 \pi}{n}}=\cos \left(\frac{2 \pi}{n}\right)+\mathbf{i} \sin \left(\frac{2 \pi}{n}\right), \mathbf{i}=\sqrt{-1}$. The well-known eigenvalue decomposition of the matrix $C_{n}=\operatorname{circ}_{n}\left(c_{0}, c_{1}, \ldots, c_{n-1}\right)$ is that

$$
C_{n}=F_{n}^{*} D_{n} F_{n}
$$

where * denotes the conjugate transpose (i.e $F_{n}^{*}=\bar{F}_{n}^{T}$ ), $F_{n}$ called $n \times n$ Fourier matrix that contains the eigenvectors of $C_{n}$ such that

$$
\left[F_{n}^{*}\right]_{u, v}=\frac{1}{\sqrt{n}} \omega^{(u-1)(v-1)}, \quad 1 \leq u, v \leq n
$$

and $D_{n}=\operatorname{diag}\left(\lambda_{1}, \lambda_{2}, \ldots, \lambda_{n}\right)$ with

$$
\lambda_{k}=\sum_{r=1}^{n} c_{r-1} \omega^{(k-1)(r-1)}, \quad 1 \leq k \leq n
$$


are the eigenvalues of $C_{n}$ [14].

It can be easily seen that the matrices $F_{n}$ and $F_{n}^{*}$ are symmetric:

$$
F_{n}=F_{n}^{T}, F_{n}^{*}=\left(F_{n}^{*}\right)^{T}=\overline{F_{n}}
$$

where we can deduce $\left[F_{n}\right]_{u, v}=\left[F_{n}\right]_{v, u}$. It is also one of fundamental property that the matrix $F_{n}$ is unitary: $F_{n} F_{n}^{*}=F_{n}^{*} F_{n}=I$ [14].

In [15], we have the eigenvector $f^{(k)}$ of $C_{n}$ corresponding to the eigenvalue $\lambda_{k}$ in (2.2) as the following

$$
f^{(k)}=\frac{1}{\sqrt{n}}\left(1, \omega^{k-1}, \omega^{2(k-1)}, \ldots, \omega^{(n-1)(k-1)}\right)^{T} .
$$

Since the product of two circulant matrices is again a circulant matrix, the $r^{\text {th }}$ power $(r \in \mathbb{N})$ of $C_{n}$ is also circulant and it is, from the well-known expression (2.1), obtained as

$$
C_{n}^{r}=F_{n}^{*} D_{n}^{r} F_{n}=F_{n}^{*} \operatorname{diag}\left(\lambda_{1}^{r}, \lambda_{2}^{r}, \ldots, \lambda_{n}^{r}\right) F_{n} .
$$

If $C_{n}$ is nonsingular, then the expression (2.5) applies to negative integers.

Definition 2.1. The Chebyshev polynomial $T_{n}(x)$ of the first kind is a polynomial in $x$ of degree $n$, defined by the relation

$$
T_{n}(x)=\cos n \theta \quad \text { when } x=\cos \theta .
$$

Definition 2.2. The Chebyshev polynomial $U_{n}(x)$ of the second kind is a polynomial of degree $n$ in $x$ defined by

$$
U_{n}(x)=\sin (n+1) \theta / \sin \theta \quad \text { when } x=\cos \theta .
$$

One can reach the following result about Chebyshev polynomials in [16]:

Let $T_{k}(x)$ and $U_{k}(x) \quad(k \in \mathbb{N} \cup\{0\})$ be the $k^{\text {th }}$ degree Chebyshev polynomials of the first and second kind, respectively. Then

$$
T_{k}(x)=\cos (k \arccos x) \text { and } U_{k}(x)=\frac{\sin ((k+1) \arccos x)}{\sin (\arccos x)}
$$

for $-1 \leq x \leq 1$. Moreover, one can find more applications related this polynomials in [17]-[19].

\section{Eigenvalues and eigenvectors of circulant $m$-diagonal matrix}

In this part, we give the expressions of eigenvalues and eigenvectors of $A_{n}=\operatorname{circ}_{n}\left(a_{0}, a_{1}, \ldots, a_{m}, 0, \ldots, 0, a_{-m}, \ldots, a_{-1}\right)$ depending on Chebyshev polynomials of the first and second kind.

Lemma 3.1. Consider $3 \leq n \in \mathbb{N}, 1 \leq m \leq\left\lfloor\frac{n-1}{2}\right\rfloor$ and $a_{i} \in \mathbb{R}(i=0, \pm 1, \ldots \pm m)$. Let $A_{n}=\operatorname{circ}_{n}\left(a_{0}, a_{1}, \ldots, a_{m}, 0, \ldots, 0, a_{-m}, \ldots, a_{-1}\right)$ be an $n \times n$ circulant matrix and $\alpha_{k}=\cos \frac{2 \pi(k-1)}{n}$ for every $1 \leq k \leq n$. Then the eigenvalues of $A_{n}$ are

$$
\lambda_{k}=a_{0}+\sum_{l=1}^{m}\left(\left(a_{l}+a_{-l}\right) T_{l}\left(\alpha_{k}\right)+\mathbf{i}\left(a_{l}-a_{-l}\right) \operatorname{sgn}\left(\frac{n}{2}+1-k\right) \sqrt{1-\alpha_{k}^{2}} \lim _{j \rightarrow k} U_{l-1}\left(\alpha_{j}\right)\right)
$$

where $\lambda_{k}$ is the $k^{\text {th }}$ eigenvalue of $A_{n}$ and $\operatorname{sgn}$ denotes the signum function.

Proof. Taking into account (2.2), (1.3) and $\omega^{(k-1)(n+2-r-1)}=\omega^{-(k-1)(r-1)}$ for all $2 \leq r \leq n$ (see [10]), we can write $\lambda_{k}$ as

$$
\begin{aligned}
\lambda_{k} & =a_{0}+a_{1} \omega^{(k-1) 1}+\ldots+a_{m} \omega^{(k-1) m}+a_{n-m} \omega^{(k-1)(n-m)}+\ldots+a_{n-1} \omega^{(k-1)(n-1)} \\
& =a_{0}+a_{1} \omega^{(k-1)}+\ldots+a_{m} \omega^{(k-1) m}+a_{-m} \omega^{-(k-1) m}+\ldots+a_{-1} \omega^{-(k-1)} .
\end{aligned}
$$

From the definition of $\omega$, we get

$$
\lambda_{k}=a_{0}+\sum_{l=1}^{m}\left(\left(a_{l}+a_{-l}\right) \cos \frac{2 \pi(k-1) l}{n}+\mathbf{i}\left(a_{l}-a_{-l}\right) \sin \frac{2 \pi(k-1) l}{n}\right) .
$$

Observe that from (2.6), we have

$$
T_{m}\left(\cos \frac{2 \pi(k-1)}{n}\right)=\cos \frac{2 \pi(k-1) m}{n},
$$

and

$$
U_{m-1}\left(\cos \frac{2 \pi(k-1)}{n}\right)=\frac{\sin \frac{2 \pi(k-1) m}{n}}{\sin \frac{2 \pi(k-1)}{n}}
$$


where there exists indeterminate form $0 / 0$ for $k=1$ and $k=\frac{n}{2}+1$. Then we can construct the expression (3.2) as

$$
\lambda_{k}=a_{0}+\sum_{l=1}^{m}\left(\left(a_{l}+a_{-l}\right) T_{l}\left(\cos \frac{2 \pi(k-1)}{n}\right)+\mathbf{i}\left(a_{l}-a_{-l}\right) \sin \frac{2 \pi(k-1)}{n} \lim _{j \rightarrow k} U_{l-1}\left(\cos \frac{2 \pi(j-1)}{n}\right)\right) .
$$

Consequently, we reach the desired result by transforming $\cos \frac{2 \pi(k-1)}{n}=\alpha_{k}$ and then

$$
\sin \frac{2 \pi(k-1)}{n}=\left\{\begin{array}{cl}
\sqrt{1-\alpha_{k}^{2}} & \text { if } \frac{n}{2}+1-k>0 \\
0 & \text { if } \frac{n}{2}+1-k=0 \\
-\sqrt{1-\alpha_{k}^{2}} & \text { if } \frac{n}{2}+1-k<0
\end{array}\right.
$$

With the help of Lemma 3.1, we reach a nice result for the eigenvalues of $A_{n}=\operatorname{circ}_{n}\left(a_{0}, a_{1}, \ldots a_{m}, 0, \ldots, 0, a_{-m}, \ldots a_{-1}\right)$. Since

$$
\cos \frac{2 \pi(n+2-k-1)}{n}=\cos \frac{2 \pi(k-1)}{n}
$$

and

$$
\sin \frac{2 \pi(n+2-k-1)}{n}=-\sin \frac{2 \pi(k-1)}{n} .
$$

We obtain that $\overline{\lambda_{k}}=\lambda_{n+2-k}(2 \leq k \leq n)$ from (3.2). Clearly, if we rewrite this eigenvalues in a diagonal matrix, then

$$
\begin{array}{ll}
D_{n}=\operatorname{diag}\left(\lambda_{1}, \lambda_{2}, \ldots, \lambda_{\frac{n+1}{2}}, \overline{\lambda_{\frac{n+1}{2}}}, \ldots, \overline{\lambda_{2}}\right) & \text { if } n \text { is odd } \\
D_{n}=\operatorname{diag}\left(\lambda_{1}, \lambda_{2}, \ldots, \lambda_{\frac{n}{2}}, \lambda_{\frac{n}{2}+1}, \overline{\lambda_{n}}, \ldots, \overline{\lambda_{2}}\right) & \text { if } n \text { is even. }
\end{array}
$$

If we take $n=8$ and $m=2$ for the matrix $A_{n}$ in (1.2), then, from Lemma 3.1, we get

$$
\begin{aligned}
\lambda_{3}= & a_{0}+ \\
+ & \left(a_{1}+a_{-1}\right) \cos \frac{\pi}{2}+\mathbf{i}\left(a_{1}-a_{-1}\right) \sin \frac{\pi}{2} \\
& +\left(a_{2}+a_{-2}\right) \cos \pi+\mathbf{i}\left(a_{2}-a_{-2}\right) \sin \pi \\
= & a_{0}-a_{2}-a_{-2}+\mathbf{i}\left(a_{1}-a_{-1}\right)
\end{aligned}
$$

and

$$
\begin{aligned}
\lambda_{7}= & a_{0}+ \\
& \left(a_{1}+a_{-1}\right) \cos \frac{3 \pi}{2}+\mathbf{i}\left(a_{1}-a_{-1}\right) \sin \frac{3 \pi}{2} \\
& +\left(a_{2}+a_{-2}\right) \cos 3 \pi+\mathbf{i}\left(a_{2}-a_{-2}\right) \sin 3 \pi \\
= & a_{0}-a_{2}-a_{-2}-\mathbf{i}\left(a_{1}-a_{-1}\right)
\end{aligned}
$$

As can be seen above, $\lambda_{7}=\overline{\lambda_{3}}$.

Corollary 3.2. Consider $3 \leq n \in \mathbb{N}, 1 \leq m \leq\left\lfloor\frac{n-1}{2}\right\rfloor$ and $a_{i} \in \mathbb{R}(i=0,1, \ldots m)$. Let $B_{n}=\operatorname{circ}_{n}\left(a_{0}, a_{1}, \ldots, a_{m}, 0, \ldots, 0, a_{m}, \ldots, a_{1}\right)$ be an $n \times n$ symmetric circulant matrix and $\alpha_{k}=\cos \frac{2 \pi(k-1)}{n}$ for every $1 \leq k \leq n$. Then the eigenvalues of $B_{n}$ are

$$
\mu_{k}=a_{0}+2 \sum_{l=1}^{m} a_{l} T_{l}\left(\alpha_{k}\right)
$$

where $\mu_{k}$ is the $k^{\text {th }}$ eigenvalue of $B_{n}$.

Proof. The proof can be straightforwardly obtained from Lemma 3.1.

Since $\cos \frac{2 \pi(n+2-k-1)}{n}=\cos \frac{2 \pi(k-1)}{n}$, we can easily see that $\mu_{k}=\mu_{n+2-k} \quad(2 \leq k \leq n)$ from Corollary 3.2. Clearly, if we rewrite this eigenvalues in a diagonal matrix again, then

$$
\begin{array}{ll}
D_{n}=\operatorname{diag}\left(\mu_{1}, \mu_{2}, \ldots, \mu_{\frac{n+1}{2}}, \mu_{\frac{n+1}{2}}, \ldots, \mu_{2}\right) & \text { if } n \text { is odd } \\
D_{n}=\operatorname{diag}\left(\mu_{1}, \mu_{2}, \ldots, \mu_{\frac{n}{2}}, \mu_{\frac{n}{2}+1}, \mu_{\frac{n}{2}}, \ldots, \mu_{2}\right) & \text { if } n \text { is even. }
\end{array}
$$

If we take $n=8$ and $m=2$ for the matrix $B_{n}=\operatorname{circ}_{n}\left(a_{0}, a_{1}, \ldots a_{m}, 0, \ldots, 0, a_{m}, \ldots a_{1}\right)$, then, from Corollary 3.2, we get

$$
\mu_{3}=a_{0}+2\left(a_{1} \cos \frac{\pi}{2}+a_{2} \cos \pi\right)=a_{0}-2 a_{2}
$$


and

$$
\mu_{7}=a_{0}+2\left(a_{1} \cos \frac{3 \pi}{2}+a_{2} \cos 3 \pi\right)=a_{0}-2 a_{2}
$$

As can be seen above, $\mu_{7}=\mu_{3}$.

Now, from the expression (2.4), let us give the following result for the eigenvectors of $A_{n}=\operatorname{circ}_{n}\left(a_{0}, a_{1}, \ldots, a_{m}, 0, \ldots, 0, a_{-m}, \ldots, a_{-1}\right)$ depending on Chebyshev polynomials of the first and second kind

Theorem 3.3. Let $3 \leq n \in \mathbb{N}, 1 \leq m \leq\left\lfloor\frac{n-1}{2}\right\rfloor$ and $a_{i} \in \mathbb{R}(i=0, \pm 1, \ldots \pm m)$ and $\alpha_{k}=\cos \frac{2 \pi(k-1)}{n}$ for every $1 \leq k \leq n$. Then the eigenvector $F_{k}$ of the matrix $A_{n}=\operatorname{circ}_{n}\left(a_{0}, a_{1}, \ldots, a_{m}, 0, \ldots, 0, a_{-m}, \ldots, a_{-1}\right)$ corresponding to the eigenvalue $\lambda_{k}$ given by (3.1) is

$$
F_{k}=\left[\begin{array}{c}
T_{0}\left(\alpha_{k}\right)+\mathbf{i} \operatorname{sgn}\left(\frac{n}{2}+1-k\right) \sqrt{1-\alpha_{k}^{2}} \lim _{\mathbf{j} \rightarrow \mathbf{k}} U_{-1}\left(\alpha_{j}\right) \\
T_{1}\left(\alpha_{k}\right)+\mathbf{i} \operatorname{sgn}\left(\frac{n}{2}+1-k\right) \sqrt{1-\alpha_{k}^{2}} \lim _{\mathbf{j} \rightarrow \mathbf{k}} U_{0}\left(\alpha_{j}\right) \\
\vdots \\
T_{n-1}\left(\alpha_{k}\right)+\operatorname{isgn}\left(\frac{n}{2}+1-k\right) \sqrt{1-\alpha_{k}^{2}} \lim _{\mathbf{j} \rightarrow \mathbf{k}} U_{n-2}\left(\alpha_{j}\right)
\end{array}\right] .
$$

Proof. From (2.4), (2.6) and (3.3), the result can be easily obtained.

We must note that each one of all circulant matrices also have the eigenvectors generated by $F_{k}$ given by (3.6)

\section{Integer powers of circulant $m$-diagonal matrix}

In this part, by using the symmetric relationship between the eigenvalues in (3.4), we give the efficient expression to compute the integer power of the circulant $m$-diagonal matrix $A_{n}=\operatorname{circ}_{n}\left(a_{0}, a_{1}, \ldots, a_{m}, 0, \ldots, 0, a_{-m}, \ldots, a_{-1}\right)$ based on Chebyshev polynomials of the first and second kind such that the method is faster than any of the classical methods which find the powers of $A_{n}$ with an amount of computations.

Theorem 4.1. Consider $3 \leq n \in \mathbb{N}, 1 \leq m \leq\left\lfloor\frac{n-1}{2}\right\rfloor$ and $a_{i} \in \mathbb{R}(i=0, \pm 1, \ldots \pm m)$. Let $A_{n}=\operatorname{circ}_{n}\left(a_{0}, a_{1}, \ldots, a_{m}, 0, \ldots, 0, a_{-m}, \ldots, a_{-1}\right)$ be an $n \times n$ nonsingular circulant m-diagonal matrix and $\alpha_{k}=\cos \frac{2 \pi(k-1)}{n}$ for every $1 \leq k \leq n$. Then the $(u, v)$ th entry of $A_{n}^{r}$ is that

$$
\left[A_{n}^{r}\right]_{u, v}=\frac{1}{n}\left(S_{1}+S_{2}\right)
$$

for all $r \in \mathbb{Z}$ and $1 \leq u, v \leq n$, where $S_{1}$ and $S_{2}$ are respectively such that

$$
\begin{aligned}
S_{1}= & \sum_{k=1}^{\left\lfloor\frac{n}{2}\right\rfloor+1}\left(a_{0}+\sum_{l=1}^{m}\left(\left(a_{l}+a_{-l}\right) T_{l}\left(\alpha_{k}\right)+\mathbf{i}\left(a_{l}-a_{-l}\right) \operatorname{sgn}\left(\frac{n}{2}+1-k\right) \sqrt{1-\alpha_{k}^{2}} \lim _{\mathbf{j} \rightarrow \mathbf{k}} U_{l-1}\left(\alpha_{j}\right)\right)\right)^{r} \\
& \times\left(T_{|u-v|}\left(\alpha_{k}\right)+\mathbf{i s g n}(u-v) \operatorname{sgn}\left(\frac{n}{2}+1-k\right) \sqrt{1-\alpha_{k}^{2}} \lim _{\mathbf{j} \rightarrow \mathbf{k}} U_{|u-v|-1}\left(\alpha_{j}\right)\right)
\end{aligned}
$$

and

$$
\begin{aligned}
S_{2}= & \sum_{k=2}^{\left\lfloor\frac{n+1}{2}\right\rfloor}\left(a_{0}+\sum_{l=1}^{m}\left(\left(a_{l}+a_{-l}\right) T_{l}\left(\alpha_{k}\right)-\mathbf{i}\left(a_{l}-a_{-l}\right) \operatorname{sgn}\left(\frac{n}{2}+1-k\right) \sqrt{1-\alpha_{k}^{2}} \lim _{\mathbf{j} \rightarrow \mathbf{k}} U_{l-1}\left(\alpha_{j}\right)\right)\right)^{r} \\
& \times\left(T_{|u-v|}\left(\alpha_{k}\right)-\mathbf{i} \operatorname{sgn}(u-v) \operatorname{sgn}\left(\frac{n}{2}+1-k\right) \sqrt{1-\alpha_{k}^{2}} \lim _{\mathbf{j} \rightarrow \mathbf{k}} U_{|u-v|-1}\left(\alpha_{j}\right)\right) .
\end{aligned}
$$

Here $\lfloor x\rfloor$ and sgn denote the largest integer less than or equal to $x$ and the signum function, respectively.

Proof. By using (2.5) and (2.3), we get

$$
\begin{aligned}
{\left[A_{n}^{r}\right]_{u, v} } & =\left[F_{n}^{*} D_{n}^{r} F_{n}\right]_{u, v}=\sum_{k=1}^{n}\left[F_{n}^{*}\right]_{u, k}\left[D_{n}^{r} F_{n}\right]_{k, v}=\sum_{k=1}^{n}\left[F_{n}^{*}\right]_{u, k} \lambda_{k}^{r}\left[F_{n}\right]_{k, v} \\
& =\sum_{k=1}^{n} \lambda_{k}^{r}\left[F_{n}^{*}\right]_{u, k}\left[F_{n}\right]_{v, k}=\frac{1}{n} \sum_{k=1}^{n} \lambda_{k}^{r} \omega^{(u-1)(k-1)} \omega^{-(v-1)(k-1)}
\end{aligned}
$$


and then

$$
\left[A_{n}^{r}\right]_{u, v}=\frac{1}{n} \sum_{k=1}^{n} \lambda_{k}^{r} \omega^{(k-1)(u-v)}
$$

where $\lambda_{k}$ is already obtained as in (3.1). Since $\overline{\lambda_{k}}=\lambda_{n+2-k}, 2 \leq k \leq n$ and $\omega^{(n+2-k-1)(u-v)}=\omega^{-(k-1)(v-u)}$, the second half of the sum in (4.1) can be written as

$$
\sum_{k=\frac{n+1}{2}+1}^{n} \lambda_{k}^{r} \omega^{(k-1)(u-v)}=\sum_{k=2}^{\frac{n+1}{2}} \lambda_{n+2-k}^{r} \omega^{(n+2-k-1)(u-v)}=\sum_{k=2}^{\frac{n+1}{2}} \bar{\lambda}_{k}^{r} \omega^{-(k-1)(u-v)}
$$

for the case $n$ is odd. The same observations can be applied in the case $n$ even and the result is that

$$
\left[A_{n}^{r}\right]_{u, v}=\frac{1}{n}\left[\sum_{k=1}^{\left\lfloor\frac{n}{2}\right\rfloor+1} \lambda_{k}^{r} \omega^{(k-1)(u-v)}+\sum_{k=2}^{\left\lfloor\frac{n+1}{2}\right\rfloor} \bar{\lambda}_{k}^{r} \omega^{-(k-1)(u-v)}\right]=\frac{1}{n}\left(S_{1}+S_{2}\right) .
$$

Thus, from the expression above, we can write

$$
S_{1}=\sum_{k=1}^{\left\lfloor\frac{n}{2}\right\rfloor+1} \lambda_{k}^{r}\left(\cos \frac{2 \pi(k-1)(u-v)}{n}+\mathbf{i} \sin \frac{2 \pi(k-1)(u-v)}{n}\right)
$$

and

$$
S_{2}=\sum_{k=2}^{\left\lfloor\frac{n+1}{2}\right\rfloor} \bar{\lambda}_{k}^{r}\left(\cos \frac{2 \pi(k-1)(u-v)}{n}-\mathbf{i} \sin \frac{2 \pi(k-1)(u-v)}{n}\right) .
$$

Since, from (2.6),

$$
T_{|u-v|}\left(\cos \frac{2 \pi(k-1)}{n}\right)=\cos \frac{2 \pi(k-1)(u-v)}{n}
$$

and

$$
U_{|u-v|-1}\left(\cos \frac{2 \pi(k-1)}{n}\right)=\operatorname{sgn}(u-v) \frac{\sin \frac{2 \pi(k-1)(u-v)}{n}}{\sin \frac{2 \pi(k-1)}{n}}
$$

with indeterminate form $0 / 0$ for $k=1$ and $k=\frac{n}{2}+1$, then

$$
S_{1}=\sum_{k=1}^{\left\lfloor\frac{n}{2}\right\rfloor+1} \lambda_{k}^{r}\left(T_{|u-v|}\left(\cos \frac{2 \pi(k-1)}{n}\right)+\mathbf{i s g n}(u-v) \sin \frac{2 \pi(k-1)}{n} \lim _{\mathbf{j} \rightarrow \mathbf{k}} U_{|u-v|-1}\left(\cos \frac{2 \pi(j-1)}{n}\right)\right),
$$

and

$$
S_{2}=\sum_{k=2}^{\left\lfloor\frac{n+1}{2}\right\rfloor} \bar{\lambda}_{k}^{r}\left(T_{|u-v|}\left(\cos \frac{2 \pi(k-1)}{n}\right)-\mathbf{i s g n}(u-v) \sin \frac{2 \pi(k-1)}{n} \lim _{\mathbf{j} \rightarrow \mathbf{k}} U_{|u-v|-1}\left(\cos \frac{2 \pi(j-1)}{n}\right)\right) .
$$

The theorem follows by substituting $\lambda_{k}$ in (3.1) and $\cos \frac{2 \pi(k-1)}{n}=\alpha_{k}$ into the above expressions.

From (2.5), we have the $r^{\text {th }}$ power of any (symmetric) circulant matrix is also a (symmetric) circulant matrix.

Consider $a_{i} \in \mathbb{R}(i=0, \pm 1)$ and let $A_{4}=\operatorname{circ}_{4}\left(a_{0}, a_{1}, 0, a_{-1}\right)$ be circulant tridiagonal matrix. Then, by using Theorem 4.1, we get $A_{4}^{r}=\operatorname{circ}_{4}\left(\tau_{0}, \tau_{1}, \tau_{2}, \tau_{3}\right)$ with

$$
\begin{aligned}
\tau_{0} & =\frac{1}{4}\left[\left(a_{0}+a_{1}+a_{-1}\right)^{r}+\left(a_{0}-\left(a_{1}+a_{-1}\right)\right)^{r}+z^{r}+\bar{z}^{r}\right], \\
\tau_{1} & =\frac{1}{4}\left[\left(a_{0}+a_{1}+a_{-1}\right)^{r}-\left(a_{0}-\left(a_{1}+a_{-1}\right)\right)^{r}-\mathbf{i} z^{r}+\mathbf{i} \bar{z}^{r}\right], \\
\tau_{2} & =\frac{1}{4}\left[\left(a_{0}+a_{1}+a_{-1}\right)^{r}+\left(a_{0}-\left(a_{1}+a_{-1}\right)\right)^{r}-\mathbf{i} z^{r}-\mathbf{i} \bar{z}^{r}\right], \\
\tau_{3} & =\frac{1}{4}\left[\left(a_{0}+a_{1}+a_{-1}\right)^{r}-\left(a_{0}-\left(a_{1}+a_{-1}\right)\right)^{r}+\mathbf{i} z^{r}-\mathbf{i} \bar{z}^{r}\right]
\end{aligned}
$$

where $z=a_{0}+\mathbf{i}\left(a_{1}-a_{-1}\right)$.

If we take $m=1$ in Theorem 4.1, the expression given in [12, Theorem 2.1] can be easily seen.

Theorem 4.1 allows us to significantly reduce the computing process while finding the integer powers of the circulant $m$-diagonal matrix $A_{n}=\operatorname{circ}_{n}\left(a_{0}, a_{1}, \ldots, a_{m}, 0, \ldots, 0, a_{-m}, \ldots, a_{-1}\right)$ 
Theorem 4.2. Consider $3 \leq n \in \mathbb{N}, 1 \leq m \leq\left\lfloor\frac{n-1}{2}\right\rfloor$ and $a_{i} \in \mathbb{R}(i=0,1, \ldots m)$. Let $B_{n}=\operatorname{circ}_{n}\left(a_{0}, a_{1}, \ldots, a_{m}, 0, \ldots, 0, a_{m}, \ldots, a_{1}\right)$ be an $n \times n$ nonsingular symmetric circulant m-diagonal matrix and $\alpha_{k}=\cos \frac{2 \pi(k-1)}{n}$ for every $1 \leq k \leq n$. Then the $(u, v)$ th entry of $B_{n}^{r}$ is that

$$
\left[B_{n}^{r}\right]_{u, v}=\frac{1}{n} \sum_{k=1}^{\left\lfloor\frac{n}{2}\right\rfloor+1} l_{n-2 k+2}\left(a_{0}+2 \sum_{l=1}^{m} a_{l} T_{l}\left(\alpha_{k}\right)\right)^{r} T_{|u-v|}\left(\alpha_{k}\right)
$$

where $\lfloor x\rfloor$ denotes the largest integer less than or equal to $x$ and

$$
l_{s}=\left\{\begin{array}{l}
1 \quad \text { if } s \in\{0, n\} \\
2 \text { in other cases. }
\end{array}\right.
$$

Proof. By using Theorem 4.1, we get

$$
S_{1}=\sum_{k=1}^{\left\lfloor\frac{n}{2}\right\rfloor+1}\left(a_{0}+2 \sum_{l=1}^{m} a_{l} T_{l}\left(\alpha_{k}\right)\right)^{r} T_{|u-v|}\left(\alpha_{k}\right)+\mathbf{i s g n}(u-v) \operatorname{sgn}\left(\frac{n}{2}+1-k\right) \sqrt{1-\alpha_{k}^{2}} U_{|u-v|-1}\left(\alpha_{k}\right)
$$

and

$$
S_{2}=\sum_{k=2}^{\left\lfloor\frac{n+1}{2}\right\rfloor}\left(a_{0}+2 \sum_{l=1}^{m} a_{l} T_{l}\left(\alpha_{k}\right)\right)^{r} T_{|u-v|}\left(\alpha_{k}\right)-\mathbf{i s g n}(u-v) \operatorname{sgn}\left(\frac{n}{2}+1-k\right) \sqrt{1-\alpha_{k}^{2}} U_{|u-v|-1}\left(\alpha_{k}\right)
$$

Since

$$
\begin{cases}\left\lfloor\frac{n+1}{2}\right\rfloor=\frac{n+1}{2} \text { and }\left\lfloor\frac{n}{2}\right\rfloor+1=\frac{n+1}{2} & \text { if } n \text { is odd } \\ \left\lfloor\frac{n+1}{2}\right\rfloor=\frac{n}{2} \text { and }\left\lfloor\frac{n}{2}\right\rfloor+1=\frac{n}{2}+1 & \text { if } n \text { is even. }\end{cases}
$$

Then

$$
\begin{aligned}
{\left[B_{n}^{r}\right]_{u, v}=} & \frac{1}{n}\left(S_{1}+S_{2}\right) \\
= & \left\{\begin{array}{cc}
\frac{1}{n}\left[\left(a_{0}+2 \sum_{l=1}^{m} a_{l}\right)^{r}+2 \sum_{k=2}^{\frac{n+1}{2}}\left(a_{0}+2 \sum_{l=1}^{m} a_{l} T_{l}\left(\alpha_{k}\right)\right)^{r} T_{|u-v|}\left(\alpha_{k}\right)\right] & \text { if } n \text { is odd, } \\
\frac{1}{n}\left[\left(a_{0}+2 \sum_{l=1}^{m} a_{l}\right)^{r}+2 \sum_{k=2}^{\frac{n}{2}}\left(a_{0}+2 \sum_{l=1}^{m} a_{l} T_{l}\left(\alpha_{k}\right)\right)^{r} T_{|u-v|}\left(\alpha_{k}\right)+\right. & \\
\left.\left(a_{0}+2 \sum_{l=1}^{m} a_{l} T_{l}\left(\alpha_{\frac{n}{2}+1}\right)\right)^{r} T_{|u-v|}\left(\alpha_{\frac{n}{2}+1}\right)\right] & \text { if } n \text { is even. }
\end{array}\right.
\end{aligned}
$$

Therefore,

$$
\left[B_{n}^{r}\right]_{u, v}=\frac{1}{n} \sum_{k=1}^{\left\lfloor\frac{n}{2}\right\rfloor+1} l_{n-2 k+2}\left(a_{0}+2 \sum_{l=1}^{m} a_{l} T_{l}\left(\alpha_{k}\right)\right)^{r} T_{|u-v|}\left(\alpha_{k}\right)
$$

which is desired.

Consider $a_{i} \in \mathbb{R}(i=0,1,2)$ and let $B_{5}=\operatorname{circ}_{5}\left(a_{0}, a_{1}, a_{2}, a_{2}, a_{1}\right)$ be a symmetric circulant pentadiagonal matrix. Then, from Theorem 4.2, we get $B_{5}^{r}=\operatorname{circ}_{4}\left(\tau_{0}, \tau_{1}, \tau_{2}, \tau_{2}, \tau_{1}\right)$ with

$$
\begin{aligned}
\tau_{0} & =\frac{1}{5}\left[\left(a_{0}+2 a_{1}+2 a_{2}\right)^{r}+2\left(a_{0}+\frac{1}{\phi} a_{1}-\phi a_{2}\right)^{r}+2\left(a_{0}-\phi a_{1}+\frac{1}{\phi} a_{2}\right)^{r}\right], \\
\tau_{1} & =\frac{1}{5}\left[\left(a_{0}+2 a_{1}+2 a_{2}\right)^{r}+\frac{1}{\phi}\left(a_{0}+\frac{1}{\phi} a_{1}-\phi a_{2}\right)^{r}-\phi\left(a_{0}-\phi a_{1}+\frac{1}{\phi} a_{2}\right)^{r}\right], \\
\tau_{2} & =\frac{1}{5}\left[\left(a_{0}+2 a_{1}+2 a_{2}\right)^{r}-\phi\left(a_{0}+\frac{1}{\phi} a_{1}-\phi a_{2}\right)^{r}+\frac{1}{\phi}\left(a_{0}-\phi a_{1}+\frac{1}{\phi} a_{2}\right)^{r}\right]
\end{aligned}
$$

where $\phi$ denotes the golden ratio.

Now, if we consider

$$
\begin{cases}m=\left\lfloor\frac{n-1}{2}\right\rfloor=\frac{n-1}{2} & \text { for } n \text { is odd, } \\ m=\left\lfloor\frac{n-1}{2}\right\rfloor+1=\frac{n}{2} & \text { for } n \text { is even, }\end{cases}
$$

in the symmetric circulant $m$-diagonal matrix $B_{n}=\operatorname{circ}_{n}\left(a_{0}, a_{1}, \ldots, a_{m}, 0, \cdots, 0, a_{m}, \cdots, a_{1}\right)$ and $a_{\frac{n}{2}} \neq 0$, then we get the symmetric circulant matrix in (1.1) discussed by Gutiérrez in [10]. And so, with the help of Theorem 4.2, we can straightforwardly reach the expression obtained by Gutiérrez in [10, Theorem 1] for positive integer powers of the matrix $B_{n}$ in (1.1). 


\section{Illustrative examples}

In this part, we give some illustrative examples. We will utilize Maple software in our calculations.

Example 5.1. Let $A_{6}=\operatorname{circ}_{6}(5,4,9,0,8,-2)$ be a circulant pentadiagonal matrix. we find the eigenvalues of $A_{6}$ by using (3.1) as

$$
\begin{array}{ll}
\lambda_{1} & =24, \\
\lambda_{2} & =-2,5000+6,0621 \mathbf{i} \\
\lambda_{3} & =-4,5000+4,3301 \mathbf{i} \\
\lambda_{4} & =20, \\
\lambda_{5}=\overline{\lambda_{3}} & =-4,5000-4,3301 \mathbf{i} \\
\lambda_{6}=\overline{\lambda_{2}} & =-2,5000-6,0621 \mathbf{i}
\end{array}
$$

and from Theorem 4.1, the entries of $A_{6}^{3}$ as

$$
A_{6}^{3}=\operatorname{circ}_{6}(3778,1008,3483,938,3651,966) .
$$

Example 5.2. Let $A_{9}=\operatorname{circ}_{9}(-2,3,-4,9,0,0,6,5,-1)$ be a circulant heptadiagonal matrix. we find the eigenvalues of $A_{9}$ by using (3.1) as

$$
\begin{array}{ll}
\lambda_{1} & =16, \\
\lambda_{2} & =-7,7942-3,6940 \mathbf{i}, \\
\lambda_{3} & =-10,0923-1,73701 \mathbf{i}, \\
\lambda_{4} & =11,5000+11,2583 \mathbf{i}, \\
\lambda_{5} & =-10,6133+9,7512 \mathbf{i}, \\
\lambda_{6}=\overline{\lambda_{5}} & =-10,6133-9,7512 \mathbf{i}, \\
\lambda_{7}=\overline{\lambda_{4}} & =11,5000-11,2583 \mathbf{i}, \\
\lambda_{8}=\overline{\lambda_{3}} & =-10,0923+1,73701 \mathbf{i}, \\
\lambda_{9}=\overline{\lambda_{2}} & =-7,7942+3,6940 \mathbf{i}
\end{array}
$$

and from Theorem 4.1, the entries of $A_{9}^{4}$ as

$$
A_{9}^{4}=\operatorname{circ}_{9}(-15410,26041,7866,-5401,16331,13209,-2024,3458,21466) .
$$

Example 5.3. Let $B_{7}=\operatorname{circ}_{7}(1,-3,2,0,0,2,-3)$ be a symmetric circulant pentadiagonal matrix. we find the eigenvalues of $B_{7}$ by using (3.5) as

$$
\begin{array}{ll}
\lambda_{1} & =-1, \\
\lambda_{2} & =-3,6310, \\
\lambda_{3} & =-1,2687, \\
\lambda_{4} & =8,8987, \\
\lambda_{5}=\lambda_{4} & =8,8987, \\
\lambda_{6}=\lambda_{3} & =-1,2687, \\
\lambda_{7}=\lambda_{2} & =-3,6310
\end{array}
$$

and from Theorem 4.2, the entries of $B_{7}^{5}$ as

$$
B_{7}^{5}=\operatorname{circ}_{7}(15771,-14485,9987,-3388,-3388,9987,-14485) .
$$

Examples 5.1, 5.2 and 5.3 can be also confirmed by means of Maple procedures given by Appendix A and B.

Appendix A. Following Maple procedure firstly generates a $n \times n$ circulant heptadiagonal matrix $A_{n}=\operatorname{circ}_{n}\left(a_{0}, a_{1}, a_{2}, a_{3}, 0, \ldots, 0\right.$, $\left.a_{-3}, a_{-2}, a_{-1}\right)$ and then compute eigenvalues and the $k^{\text {th }}$ power $(r \in \mathbb{Z})$ of it.

restart:

with(LinearAlgebra):

m:='3':

$\mathrm{n}:=$ 'n':

r:='r':

$\mathrm{a}[0]:=' \mathrm{a}[0] '$ :

$\mathrm{a}[1]:=' \mathrm{a}[1]$ ':

$\mathrm{a}[-1]:=' \mathrm{a}[-1]^{\prime}:$

$\mathrm{a}[2]:=' a[2] '$ :

$\mathrm{a}[-2]:=' \mathrm{a}[-2]$ ' : 
$\mathrm{a}[3]:=' \mathrm{a}[3] '$ :

$\mathrm{a}[-3]:=' \mathrm{a}[-3] ':$

$\mathrm{f}:=(\mathrm{i}, \mathrm{j}) \rightarrow \operatorname{piecewise}(\mathrm{i}=\mathrm{j}, \mathrm{a}[0], \mathrm{j}>\mathrm{i}$ and $\mathrm{j}-\mathrm{i}<\mathrm{m}+1, \mathrm{a}[\mathrm{j}-\mathrm{i}], \mathrm{i}>\mathrm{j}$ and $\mathrm{i}-\mathrm{j}<\mathrm{m}+1, \mathrm{a}[\mathrm{j}-\mathrm{i}], \mathrm{n}-\mathrm{j}+\mathrm{i}<\mathrm{m}+1, \mathrm{a}[\mathrm{j}-\mathrm{i}-\mathrm{n}], \mathrm{n}-\mathrm{i}+\mathrm{j}<\mathrm{m}+1, \mathrm{a}[\mathrm{j}-\mathrm{i}+\mathrm{n}])$ :

$\mathrm{A}[\mathrm{n}]:=\operatorname{Matrix}(\mathrm{n}, \mathrm{n}, \mathrm{f})$;

alpha: $=\mathrm{k} \rightarrow$ evalf $\left(\cos \left(2 * \mathrm{Pi}^{*}(\mathrm{k}-1) / \mathrm{n}\right)\right)$ :

lambda: $=\mathrm{k} \rightarrow \operatorname{evalf}\left(\left(\mathrm{a}[0]+\operatorname{sum}\left((\mathrm{a}[\mathrm{l}]+\mathrm{a}[-1]) *\right.\right.\right.$ ChebyshevT $(1$, alpha $(\mathrm{k}))+\mathrm{I} *(\mathrm{a}[1]-\mathrm{a}[-1]) * \operatorname{signum}((\mathrm{n} / 2)+1-\mathrm{k}) * \operatorname{sqrt}\left(1-(\operatorname{alpha}(\mathrm{k}))^{\wedge} 2\right)^{*} \operatorname{limit}$ (ChebyshevU(l-1,alpha(j)),j=k),l=1..m))):

$\mathrm{g}:=(\mathrm{i}, \mathrm{j}) \rightarrow \operatorname{piecewise}(\mathrm{i}=\mathrm{j}, \operatorname{lambda}(\mathrm{i}), 0):$

$\mathrm{p}:=(\mathrm{u}, \mathrm{v}) \rightarrow \operatorname{evalf}\left((1 / \mathrm{n}) *\left(\operatorname{sum}\left(\left(\operatorname{lambda}(\mathrm{k}){ }^{\wedge} \mathrm{r}\right) *\left(\operatorname{ChebyshevT}(\operatorname{abs}(\mathrm{u}-\mathrm{v}), \operatorname{alpha}(\mathrm{k}))+\mathrm{I}^{*} \operatorname{signum}(\mathrm{u}-\mathrm{v})^{*} \operatorname{signum}((\mathrm{n} / 2)+1-\mathrm{k})^{*}\right.\right.\right.\right.$

$\left.\operatorname{sqrt}\left(1-(\operatorname{alpha}(\mathrm{k}))^{\wedge} 2\right)^{*} \operatorname{limit}(\operatorname{ChebyshevU}(\operatorname{abs}(\mathrm{u}-\mathrm{v})-1, \operatorname{alpha}(\mathrm{j})), \mathrm{j}=\mathrm{k})\right), \mathrm{k}=1$..floor $\left.(\mathrm{n} / 2)+1\right)+\operatorname{sum}\left(\operatorname{conjugate}(\operatorname{lambda}(\mathrm{k}))^{\wedge} \mathrm{r}^{*}\right.$

$\left(\right.$ ChebyshevT(abs(u-v),alpha(k))-I*signum $\left.(\mathrm{u}-\mathrm{v})^{*} \operatorname{signum}((\mathrm{n} / 2)+1-\mathrm{k}) * \operatorname{sqrt}\left(1-(\operatorname{alpha}(\mathrm{k}))^{\wedge} 2\right)^{*} \operatorname{ChebyshevU}(\mathrm{abs}(\mathrm{u}-\mathrm{v})-1, \mathrm{alpha}(\mathrm{k}))\right)$, $\mathrm{k}=2$..floor $((\mathrm{n}+1) / 2))))$ :

eigenvalues_of_A[n]:=Matrix $(\mathrm{n}, \mathrm{n}, \mathrm{g})$;

the_rth_power_of_A[n]:=Matrix(n,n,p);

Appendix B. Following Maple procedure firstly generates a $n \times n$ symmetric circulant heptadiagonal matrix $B_{n}=\operatorname{circ}_{n}\left(a_{0}, a_{1}, a_{2}\right.$, $\left.a_{3}, 0, \ldots, 0, a_{3}, a_{2}, a_{1}\right)$ and then compute eigenvalues and the $k^{t h}$ power $(r \in \mathbb{Z})$ of it.

restart:

with(LinearAlgebra):

m:='3':

n:='n':

$\mathrm{a}[0]:=' \mathrm{a}[0]$ ':

$\mathrm{a}[1]:=' \mathrm{a}[1]$ ':

$\mathrm{a}[2]:=' \mathrm{a}[2]$ ':

$\mathrm{a}[3]:=' \mathrm{a}[3]$ ':

r:='r':

$\mathrm{f}:=(\mathrm{i}, \mathrm{j}) \rightarrow$ piecewise $(\mathrm{i}=\mathrm{j}, \mathrm{a}[0], \mathrm{i}>\mathrm{j}$ and $\mathrm{i}-\mathrm{j}<\mathrm{m}+1, \mathrm{a}[\mathrm{i}-\mathrm{j}], \mathrm{i}<\mathrm{j}$ and $\mathrm{j}-\mathrm{i}<\mathrm{m}+1$,

$a[j-i], i<j$ and $j-i>n-m-1, a[n-(j-i)], i>j$ and $i-j>n-m-1, a[n-(i-j)], 0)$ :

$\mathrm{B}[\mathrm{n}]:=\operatorname{Matrix}(\mathrm{n}, \mathrm{n}, \mathrm{f})$;

alpha: $=\mathrm{k} \rightarrow \operatorname{evalf}(\cos (2 * \mathrm{Pi} *(\mathrm{k}-1) / \mathrm{n}))$ :

$\mathrm{mu}:=\mathrm{k} \rightarrow \operatorname{evalf}(\mathrm{a}[0]+2 * \operatorname{sum}(\mathrm{a}[1] *$ ChebyshevT $(1, \mathrm{alpha}(\mathrm{k})), \mathrm{l}=1 . . \mathrm{m}))$ :

$\mathrm{g}:=(\mathrm{i}, \mathrm{j}) \rightarrow \operatorname{piecewise}(\mathrm{i}=\mathrm{j}, \mu(\mathrm{i}), 0)$ :

$\mathrm{l}:=(\mathrm{s}) \rightarrow$ piecewise $(\mathrm{s}=0,1, \mathrm{~s}=\mathrm{n}, 1,2)$ :

$\mathrm{p}:=(\mathrm{u}, \mathrm{v}) \rightarrow \operatorname{evalf}\left((1 / \mathrm{n}) *\left(\left(\operatorname{sum}\left(\left(\left(1(\mathrm{n}-2 * \mathrm{k}+2) *(\mu(\mathrm{k}))^{\wedge} \mathrm{r}\right) *(\operatorname{ChebyshevT}(\operatorname{abs}(\mathrm{u}-\mathrm{v})\right.\right.\right.\right.\right.$, alpha $\left.\left.\left.\left.(\mathrm{k}))), \mathrm{k}=1 . . f(\operatorname{loor}(\mathrm{n} / 2)+1)\right)\right)\right)\right)$ :

eigenvalues_of_B[n]:=Matrix $(\mathrm{n}, \mathrm{n}, \mathrm{g})$;

the_rt_power_of_B[n]:=Matrix $(\mathrm{n}, \mathrm{n}, \mathrm{p})$;

\section{Conclusion}

There has been recently increasing research interest in circulant matrices in several areas, such as digital signal processing, image compression, physics/engineering simulations, number theory, coding theory, cryptography, and, naturally, linear algebra. This paper present eigenvalues, eigenvectors, powers of circulant $m$-diagonal matrix which is one type of circulant matrices by using some famous relations on chebyshev polynomials.

\section{Acknowledgement}

The author would like to thank the anonymous referees for their careful reading of the paper and very detailed proposals that helped improve the presentation of the paper.

\section{References}

[1] N. L. Tsitsas, E.G. Alivizatos, G.H. Kalogeropoulos, A recursive algorithm for the inversion of matrices with circulant blocks, Appl. Math. Comput., 188(1) (2007), 877-894.

[2] G. Zhao, The improved nonsingularity on the r-circulant matrices in signal processing, International Conference On Computer Techology and Development, Kota Kinabalu, (ICCTD 2009), (2009), 564-567.

[3] W. Zhao, The inverse problem of anti-circulant matrices in signal processing, Pacific-Asia Conference on Knowledge Engineering and Software Engineering, Shenzhen, (KESE 2009), (2009), 47-50.

[4] J. Rimas, Investigation of dynamics of mutually synchronized systems, Telecommun. Radio Eng., 31(2) (1977), 68-79.

[5] J. Rimas, G. Leonaite, Investigation of a multidimensional automatic control system with delays and chain form structure, Inf. Technol. Control, 35(1) (2006), 65-70.

[6] J. Rimas, On computing of arbitrary positive integer powers for one type of odd order symmetric circulant matrices-I, Appl. Math. Comput., 165(1) (2005), 137-141.

[7] J. Rimas, On computing of arbitrary positive integer powers for one type of odd order symmetric circulant matrices-II, Appl. Math. Comput., 169(2) (2005), 1016-1027.

[8] J. Rimas, On computing of arbitrary positive integer powers for one type of even order symmetric circulant matrices-I, Appl. Math. Comput., 172(1) (2006), 86-90. 
[9] J. Rimas, On computing of arbitrary positive integer powers for one type of even order symmetric circulant matrices-II, Appl. Math. Comput., 174(1) (2006), 511-552

[10] J. Gutiérrez-Gutiérrez, Positive integer powers of complex symmetric circulant matrices, Appl. Math. Comput., 202(2) (2008), 877-881.

[11] F. Köken, D. Bozkurt, Positive integer powers for one type of odd order circulant matrices, Appl. Math. Comput., 217(9) (2011), 4377-4381.

[12] A. Öteleş, M. Akbulak, A Single Formula for Integer Powers of Certain Real Circulant Matrix of Odd and Even Order, Gen. Math. Notes, 35(2) (2016), 15-28.

[13] Z. Jiang, H. Xin, H. Wang, On computing of positive integer powers for r-circulant matrices, Appl. Math. Comput., 265 (2015), $409-413$.

[14] P. J. Davis, Circulant Matrices, Chelsea Publishing, New York, 1994.

[15] R. M. Gray, Toeplitz and circulant matrices: A review, Found. Trends Commun. Inform. Theory, 2(3) (2006), 155-239.

[16] J. C. Mason, D. C. Handscomb, Chebyshev Polynomials, CRC Press, Washington, 2003.

[17] C. Kızılates, N. Tuğlu, B. Cekim, On the ( $p, q)$-Chebyshev Polynomials and Related Polynomials, Mathematics, 7(136) (2019), 1-12.

[18] S. Wang, Some new identities of Chebyshev polynomials and their applications, Adv. Differ. Equ., 2015:355 (2015), 1-8.

[19] C. Cesarano, Generalized Chebyshev polynomials, Hacet. J. Math. Stat., 43(5) (2014), 731-740. 